\title{
Letter to the Editor: Perioperative Mortality Rates as a Health Metric for Acute Abdominal Surgery in Low- and Middle-Income Countries: A Systematic Review and Future Recommendations
}

\author{
Alejandro Usubillaga Villa ${ }^{1}$ Herney Andrés García-Perdomo ${ }^{1,2}$ (1)
}

Published online: 12 August 2019

(C) Société Internationale de Chirurgie 2019

Dear editor, regarding the systematic review written by Felizaire et al. [1] and published in your prestigious journal, we have found some points we would like to discuss.

First, there is no information regarding the inclusion of non-published (gray) literature and resources other than those found in the referenced databases. According to the Cochrane manual [2], this is an important and mandatory step to perform in order to avoid publication bias.

Secondly, there is no description in the methods section regarding the languages of the indexed studies included in the systematic review. When reading the protocol in PROSPERO (CRD42017060598), we found that the authors reported only articles found in the French language and disregarded all others. It is important to include referenced studies in all languages to avoid a language bias $[2,3]$. As Bartholomaus et al. [4] stated, around $90 \%$ of medical journals indexed in Medline are in English. In addition, it is important to note that there are 20 counties with Spanish as their official language and no Spanish studies must be included.

Thirdly, the research was only performed until March 2017 and no updated information was included from studies published from 2017 to 2019. This might indicate that the authors had a selection/information bias since they did not include more possible recent studies (two years of research).

Lastly, the authors reported the inclusion of another systematic review which does not agree with the correct

Herney Andrés García-Perdomo

herney.garcia@correounivalle.edu.co

1 UROGIV Research Group, Universidad del Valle, Cali, Colombia

2 Department of Surgery/Urology, School of Medicine, Universidad del Valle, Cali, Colombia methodology of systematic reviews [2]. The studies from that manuscript could have been included after analyzing whether they accomplish the inclusion criteria.

Assessing correctly the perioperative mortality rate as a health metric for acute abdominal surgery in low- and middle-income countries is impetrative due to the fact that it is a huge problem affecting those countries. For this reason, we wanted to share these important issues that require improvement, in order to enhance the quality and transparency of this and other systematic reviews [5].

\section{References}

1. Felizaire MR, Paradis T, Beckett A, Fata P, Grushka J, Johnson W et al (2019) Perioperative mortality rates as a health metric for acute abdominal surgery in low- and middle-income countries: a systematic review and future recommendations. World J Surg. https://doi.org/10.1007/s00268-019-04993-1

2. Higgins JPT (2011) GS Manual Cochrane de revisiones sistemáticas de intervenciones. Version 5.1.0 [updated March 2011]. The Cochrane Collaboration, 2011 (March):1-639

3. García-Perdomo HA (2016) Evidence synthesis and meta-analysis: a practical approach. Int J Urol Nurs 10(1):30-36

4. Bartholomäus E, Goldbeck-Wood S, Sewering M, Baethge C (2015) Experiences with bilingual publishing: surveys of authors and editors. Learn Publ 28(4):283-291

5. Garcia-Perdomo H (2018) Enhancing the quality and transparency of systematic reviews. Colomb Med 49(4):251-253

Publisher's Note Springer Nature remains neutral with regard to jurisdictional claims in published maps and institutional affiliations. 